\title{
Conocimientos, percepciones y prácticas relacionadas a VIH/SIDA en adolescentes de un distrito de Lambayeque-Perú, 2015*
}

\section{Knowledge, perceptions, and practices related to HIV/ AIDS in adolescents from a district in Lambayeque-Peru, 2015}

Correspondencia Juan William Baca Sánchez Calle Miguel Grau \#724 Urb. Campodónico, Chiclayo, Lambayeque - Perú. Teléfono: (+51) 959419819 juanwbs90@hotmail.com

Recibido: 06/08/2018

Arbitrado por pares

Aprobado: 19/12/2018

Citar como: Baca-Sánchez J, Hidalgo-Palacios C, León-Jiménez F, Malca-Tello N. Conocimientos, percepciones y prácticas relacionadas a $\mathrm{VIH} / \mathrm{SIDA}$ en adolescentes de un distrito de Lambayeque-Perú, 2015. Acta Med Peru. 2019;36(1):38-45

\author{
Juan Baca-Sánchez ${ }^{1 a}$, Claudia Hidalgo-Palacios ${ }^{1 a}$, Franco León-Jiménez ${ }^{1,2 b, c}$, \\ Nancy Malca-Tello $2 \mathrm{~d}, \mathrm{e}$ \\ 1 Universidad Católica Santo Toribio de Mogrovejo. Chiclayo, Perú. \\ 2 Hospital Regional Lambayeque. Lambayeque, Perú. \\ a Estudiante de Medicina, b Médico Internista, c Magister en epidemiologia clínica, d Bióloga, e Doctora en Bienestar social
}

\section{RESUMEN}

Objetivo: Describir los conocimientos, percepciones y prácticas relacionadas a VIH/SIDA en adolescentes de un distrito de Lambayeque-Perú en el 2015. Materiales y métodos: Estudio transversal. Se encuestaron 677 alumnos de colegios públicos y privados seleccionados mediante muestreo polietápico por conglomerados mediante un cuestionario estructurado. Se exploró asociación entre variables sociodemográficas, el conocimiento y las prácticas. Resultados: Se encuestaron 353 mujeres (52,1\%) y 324 hombres (47,9\%); 37,4\% fueron de tercero, $32,6 \%$ de cuarto y $29,9 \%$ de quinto; $61,3 \%$ fueron de colegios públicos y $38,7 \%$ de privados; $71,3 \%$ manifestaron que su conocimiento sobre VIH/SIDA era intermedio. La fuente de información más frecuente fueron las clases de colegio (68,09\%); en conocimientos, las vías de contagio más reportadas fueron: relaciones sexuales con persona infectada $(88,8 \%)$ y transfusión sanguínea $(80,9 \%)$. En los públicos se halló mayor reporte de información por internet $(p=0,019)$, que el contacto sexual con persona infectada $(p=0,007)$ y las relaciones homosexuales aumentaban el riesgo de contagio $(p=0,023)$. En los privados se halló mayor reporte de información por televisión $(p=0,081)$ y que tener una sola pareja $(p=0,013)$ y la abstinencia $(p=0,004)$, disminuían el riesgo de contagio. Los hombres refirieron un mayor riesgo de contagio en relaciones sexuales con prostitutas y homosexuales $(p=0,001)$ y $(p=0,001)$, respectivamente. Conclusiones: Las clases de colegio y el internet son importantes fuente de información sobre VIH-SIDA. Los alumnos de colegios públicos tienen mayor conocimiento en los factores de riesgo y los de colegio privado mayor conocimiento en las formas de prevención del VIH-SIDA.

Palabras clave: Conocimientos; Percepción; Conducta Social; VIH-SIDA; Conducta del Adolescente (fuente: DeCS BIREME). 


\section{ABSTRACT}

Objective: To describe knowledge, perceptions, and practices related to HIV / AIDS in adolescents from a district in Lambayeque during 2015. Materials and methods: This is a cross-sectional survey. We interviewed 677 students from public and private schools from Lambayeque, who were selected using a multi-stage complex cluster sampling. A structured questionnaire was administered. Associations between sociodemographic variables, knowledge and practices were explored. Results: Three hundred and fifty-three (52.14\%) females and 324 (47.86\%) males were interviewed. Thirty-seven (37.37\%) were from third year in high school, $32.64 \%$ were from fourth year, and $29.99 \%$ were from fifth year. Nearly two thirds of all subjects (61.30\%) were students from public schools; 483 (71.34\%) declared their knowledge on HIV/AIDS was intermediate level. The most frequently reported information source was school classes (68.09\%); with respect to knowledge, the infection routes most frequently reported were: sex with an infected person $(88.77 \%)$ and blood transfusions $(80.95 \%)$. Students from public schools declared their mostly used information source was the Web $(p=0.019)$, and they declared finding out that sexual contact with an infected person was a form of infection $(p=0.007)$ and that homosexual relationships increase the risk of infection $(p=0.023)$. Adolescents from private schools declared their most frequent information source was TV $(p=0.081)$, and they declared that having one sex partner $(p=0.013)$ and abstinence $(p=0.004)$ decreased the risk of HIV transmission. Males reported there is more risk for acquiring this infection thorough sex contact with prostitutes and homosexual men ( $p=0.001$ for both variables, respectively). Conclusions: School lectures and the Web are important information sources on HIV-AIDS. Public school adolescents have greater knowledge on risk factors, and those from private schools show better understanding on preventive measures against HIV-AIDS.

Keywords: Knowledge; Perception; Social Behavior; HIV-AIDS; Adolescent Behavior (source: MeSH NLM).

\section{INTRODUCCIÓN}

En la actualidad, el VIH/SIDA es un problema de salud pública en el Perú y a nivel mundial. En el 2013, hubo 35,3 millones de personas viviendo con VIH en todo el mundo y 1,6 millones de muertes por dicha causa ${ }^{[1]}$. En América Latina la epidemia de VIH permanece estable; por ejemplo, en el 2012, había 1,5 millones de personas, en comparación a los 1,2 millones en el 2001 , con una prevalencia estimada en adultos de $0,4 \%$. Así mismo, alrededor de 86000 personas contrajeron el VIH en el 2012, comparado con 93000 en el 2001; lo que es coherente con una disminución de un $13,3 \%$ en el número de personas que murieron por causas relacionadas con el SIDA entre los años 2005 y $2011^{[2]}$. Sin embargo, a nivel mundial todavía existen muchos retos para controlar la epidemia, teniendo en cuenta que esta se desarrolla de manera particular en cada país, con determinantes propios y una respuesta específica desde el estado y la sociedad civil ${ }^{[2]}$.

En Perú, según estimaciones de ONUSIDA hasta el año 2012, hubo 76000 casos de personas con VIH y 4100 muertes por dicha causa ${ }^{[3]}$; adicionalmente, la Dirección General de Epidemiologia del Ministerio de Salud reportó que en el periodo 1986 al 2012, 781 pacientes con VIH tuvieron entre 15-19 años ${ }^{[1]}$. Sin embargo, el comportamiento de la infección por VIH tiene una tendencia creciente en comparación con los cuatro últimos años, a expensas de los grupos poblacionales vulnerables, como son los adolescentes ${ }^{[2]}$.

En la región norte del país, el departamento de Lambayeque ocupó el sétimo lugar respecto al número de casos de VIH-SIDA en el periodo $1983-2012^{[4]}$. En este departamento existen 9,888 adolescentes de los cuales, 3,361 viven en el distrito de Ferreñafe; por lo que, los adolescentes representan el mayor grupo etario en este distrito ${ }^{[4]}$. Adicionalmente, en este distrito se presentó un incremento de casos de VIH/SIDA según reportes del año 2008 al $2011^{[2]}$.

Por ende, las estrategias de salud pública para la prevención de la infección por VIH en esta región deben buscar ser efectivas e incidir en lugares de afluencia de adolescentes, como lo son las escuelas públicas. Según la Encuesta Global de Salud Escolar realizada en el Perú, en el 2010, en alumnos de secundaria de colegios públicos, el 92,2\% había oído alguna vez de VIH/SIDA y $80 \%$ habían recibido información sobre medidas preventivas en el colegio; a pesar de ello, solo el $64 \%$ refirió que había utilizado preservativo en su última relación sexual ${ }^{[5]}$. Sin embargo, Leguía, en un estudio en adolescentes de un colegio de Chiclayo en el 2011, halló que el nivel de conocimientos sobre VIH-SIDA fue bajo en $58,3 \%$, medio en $22,7 \%$ y suficiente en $19,0 \%{ }^{[6]}$.

Los adolescentes presentan patrones comunes en sus concepciones sobre la infección por VIH-SIDA. A nivel regional, un estudio realizado en el año 2008 encontró que en este grupo etario los factores de riesgo (práctica sexual sin protección y de contacto con los grupos de población específicos), las consecuencias (muerte física y social, siendo este último entendido como el rechazo social de las personas que viven con el VIH / SIDA) y la prevención de enfermedades (basado en la información y el uso del preservativo) son elementos fundamentales en la conceptualización de la enfermedad ${ }^{[7]}$.

Dado el incremento del número de casos en el distrito y la ausencia de datos locales actualizados, se realizó el presente estudio, con el objetivo de describir los conocimientos, percepciones y prácticas relacionados a VIH/SIDA en adolescentes del distrito de Ferreñafe en el año 2015 y explorar su asociación con variables sociodemográficas. 


\section{MATERIALES Y MÉTODOS}

\section{Diseño y población del estudio}

Estudio Descriptivo transversal realizado en el distrito de Ferreñafe, ubicado en el departamento de Lambayeque al norte del Perú. Se incluyeron 1724 estudiantes del $3^{\circ}, 4^{\circ}$ y $5^{\circ}$ año de secundaria del distrito de Ferreñafe en el año 2015, quienes debían cumplir con tener la condición de residentes (vivir al menos 15 días del mes en Ferreñafe), firma del consentimiento informado del padre o tutor y asentimiento por parte del participante. Se excluyeron a aquellos estudiantes que no pudieran comprender el cuestionario, o que hablaran un idioma principal diferente al español.

\section{Muestra y muestreo}

Muestreo complejo polietápico por conglomerados. Los dos estratos iniciales fueron: colegio público y privado. En el distrito existen dos colegios públicos y 6 privados, siendo la relación entre el número de alumnos de público/privado de 2 a 1 . Se eligió un colegio público y tres privados aleatoriamente; la segunda unidad de muestreo fueron las secciones de cada año. La unidad de análisis fue el alumno elegido aleatoriamente en cada sección. EI tamaño de muestra calculado con Epidat v3.1, fue 630 alumnos, teniendo en cuenta una población de 1724 , una proporción esperada de conocimiento adecuado en relación a VIH/SIDA de $50 \%$ (ausencia de dato previo), un nivel de confianza del $95 \%$, una precisión del $5 \%$ y un efecto de diseño de 2 .

\section{Instrumento de recolección de datos}

Se aplicó un cuestionario estructurado para medir conocimientos, percepciones y prácticas sobre VIH/SIDA, tomando como referencia el utilizado en un estudio sobre conocimientos sobre VIH/SIDA en un colegio de Chiclayo durante el año $2011^{[6]}$. Luego de autorizado su uso, se seleccionaron y modificaron algunas preguntas y luego de la revisión bibliográfica se incluyeron otras preguntas. Fue revisado por siete expertos: epidemiólogo (2), médico internista (2), salubrista (1), infectólogo (1) y biólogo (1), quienes modificaron la sintaxis y el contenido de algunas preguntas. Se aplicó una prueba piloto a 52 estudiantes de secundaria de Ferreñafe para identificar problemas de inteligibilidad y medir tiempos. El cuestionario final fue autoadministrado; se distribuyó en cuatro partes: datos sociodemográficos, conocimientos (11 preguntas), percepciones (4 preguntas) y prácticas (4 preguntas), de alternativa múltiple. Tres preguntas fueron abiertas, calificándose como correctas luego de la evaluación por un médico internista. Cada pregunta del cuestionario fue calificada independientemente de la otra y se calificó la frecuencia de acierto de cada pregunta. La aplicación del cuestionario duró 20 minutos.

El recojo de la información fue realizado por los autores de este estudio, previamente entrenados por un epidemiólogointernista. Se explicaron los objetivos del estudio y el cuestionario fue administrado al término de clases.
Para evitar el sesgo de información, los autores respetaron la elección de alumnos de manera aleatoria en cada sección y el cuestionario fue administrado un solo día por colegio, de manera simultánea por cada sección, teniendo un lapso de tiempo de aproximadamente dos horas por cada grado, dentro de las cuales, ninguno de ellos tuvo contacto con otras secciones.

Para la evaluación de los datos obtenidos en el cuestionario, pasó por revisión de los expertos que participaron inicialmente en su elaboración, mencionados anteriormente. Se consideró necesaria esta revisión por expertos, ya que el cuestionario cuenta con preguntas no solo de alternativa múltiple, sino también con preguntas abiertas.

\section{Análisis estadístico}

Se calcularon frecuencias y porcentajes para variables categóricas y desviación estándar, media y mediana para la edad. Se calificó la frecuencia de acierto de cada pregunta del cuestionario. Se realizó un análisis exploratorio para buscar asociación entre las variables sociodemográficas y las preguntas del cuestionario. Finalmente se realizó un análisis exploratorio multivariado mediante modelos lineales generalizados con ayuda del software STATA v. $12.0^{\circledR}$ (Stata Corp., College Station, TX, US). Se obtuvieron razones de prevalencia (RP), e intervalos de confianza del $95 \%$ (IC 95\%); así mismo, se consideraron diferencias estadísticas a un nivel de significancia de 0,05.

\section{Aspectos éticos}

Se obtuvo el consentimiento de los padres y el asentimiento informado de los participantes previamente al inicio de la participación en el estudio. Se mantuvo el anonimato durante el llenado del cuestionario, y los datos fueron codificados garantizando la confidencialidad de las identidades de los participantes. El trabajo de investigación fue revisado y aprobado por el Comité de Ética en Investigación (CIE) de la Facultad de Medicina de la Universidad Católica Santo Toribio de Mogrovejo.

\section{RESULTADOS}

Se abordaron 700 estudiantes, aceptaron participar 677 (96,7\%). Hubo 353 mujeres $(52,1 \%$ ) y 324 hombres $(47,9 \%)$; la media de edad en mujeres fue de 15,1 $\pm 0,2$ años y en hombres, 15,1 $\pm 0,2$ años; con un rango de edades de 13 a 19 años. Según años de estudio, hubo $253(37,4 \%)$ estudiantes de tercero de secundaria, 221 (32,6\%) de cuarto y 203 (29,9\%) de quinto; 415 estudiantes $(61,3 \%)$ fueron de colegios públicos y $262(38,7 \%)$ de colegios privados.

El 59,2\% y $38,3 \%$ refirió saber que significaban la palabra "SIDA" y "VIH", respectivamente. De éstos, sólo 49,1\% y 34,7\% definieron correctamente las siglas de dichas palabras, respectivamente. El 27,3\%, del total de encuestados, refirió que "no existe diferencia entre tener VIH o SIDA". Sólo el 40,5\% de los que mencionaron que había diferencia, la explicó correctamente. 
Tabla 1. Conocimientos y percepciones sobre VIH/SIDA en adolescentes de un distrito de Lambayeque-Perú, 2015.

\section{Variable de estudio}

N $\%$

Percepción de conocimientos sobre VIH-SIDA

$\begin{array}{lcc}\text { Medio } & 483 & 71,3 \\ \text { Bajo } & 116 & 17,1 \\ \text { Alto } & 73 & 0,8 \\ \text { En blanco } & 5 & 0,7\end{array}$

Recibieron información sobre VIH/SIDA en el colegio

$\begin{array}{lcc}\text { Sí } & 566 & 83,6 \\ \text { No } & 83 & 12,3 \\ \text { No sé } & 25 & 3,7 \\ \text { En blanco } & 3 & 0,4\end{array}$

EI VIH-SIDA tiene cura

$\begin{array}{lcc}\text { No tiene cura } & 462 & 68,2 \\ \text { Si tiene cura } & 104 & 15,4 \\ \text { No sé } & 104 & 15,4 \\ \text { En blanco } & 7 & 1,0\end{array}$

Persona asintomática con VIH-SIDA puede contagiar

$\begin{array}{lcc}\text { Sí } & 429 & 63,4 \\ \text { No sé } & 171 & 25,3 \\ \text { No } & 68 & 10,0 \\ \text { En blanco } & 9 & 1,3\end{array}$

Protección total del preservativo

$\begin{array}{lcc}\text { No } & 482 & 71,2 \\ \text { No sé } & 101 & 14,9 \\ \text { Sí } & 91 & 13,4 \\ \text { En blanco } & 3 & 0,4\end{array}$

Medio de obtención de información acerca del VIH/SIDA

$\begin{array}{lcc}\text { Clases del colegio } & 461 & 68,1 \\ \text { Internet } & 302 & 44,6 \\ \text { Televisión } & 259 & 38,3 \\ \text { Padres } & 255 & 37,7 \\ \text { Amigos } & 108 & 15,9 \\ \text { Otros } & 44 & 6,5\end{array}$

Vías de contagio

Relaciones sexuales con infectado $\quad 60188,8$

Recibir transfusión sanguínea $\quad 548 \quad 80,9$

$\begin{array}{lll}\text { Pincharse con aguja utilizada } & 520 \quad 76,8\end{array}$

$\begin{array}{lll}\text { Picadura de mosquito } & 97 & 14,3\end{array}$

Compartir utensilios de cocina $\quad 75 \quad 11,1$

Beso en la boca $\quad 73 \quad 10,8$

Medidas preventivas de contagio

$\begin{array}{lcc}\text { Tener una sola pareja } & 442 & 65,3 \\ \text { Evitar tener relaciones con prostitutas } & 440 & 64,9 \\ \text { Usar preservativo } & 394 & 58,2 \\ \text { Evitar relaciones homosexuales } & 365 & 53,9 \\ \text { Abstinencia } & 242 & 35,7 \\ \text { Coitus interruptus } & 84 & 12,4 \\ \text { Otro } & 19 & 2,8\end{array}$

El 71,3\% manifestó tener un nivel intermedio de conocimientos sobre VIH/SIDA; $68,1 \%$ mencionó que la principal fuente de información fue el colegio; sin embargo, el 83,6\% de ellos refirió que habían recibido clases sobre VIH/SIDA en el colegio; $68,2 \%$ reportaron creer, que el "SIDA no tiene cura"; $58 \%$ referían que el preservativo es una medida preventiva y $13,4 \%$ del total de encuestados referían que su uso brinda protección absoluta. La forma de contagio más reportada fue: tener relaciones sexuales con persona infectada $(88,8 \%)$ y la medida más efectiva para disminuir el contagio fue: tener una sola pareja (65,3\%); $12,4 \%$ del total creían que el "coitus interruptus" era una medida efectiva para prevenir el contagio (Tabla 1).

En cuanto a prácticas, $17,3 \%$ refirió haber tenido relaciones sexuales ( $26,8 \%$ hombres y $8,5 \%$ mujeres), mientras que el $90,8 \%$ manifestó que utilizaría preservativo en un encuentro sexual con una persona desconocida (Tabla 2).

Al comparar tipos de colegio, los alumnos que provenían de escuelas públicas refirieron disminución de contagio si se evitaban relaciones con homosexuales $(p=0,023)$, mayor reporte de información por internet $(p=0,019)$ y que el contacto sexual con persona infectada, aumentaba el riesgo de contagio $(p=0,007)$. Con mayor frecuencia opinaban que la enfermedad tiene cura $(p=0,012)$.

Por otro lado, los alumnos de colegios privados mencionaron a la televisión como la principal fuente de información $(p=0,019)$, que la vía principal vía de infección son las relaciones sexuales $(p=0,007)$ y que la abstinencia sexual y tener una sola pareja

Tabla 2. Características de las prácticas sexuales referidas relacionadas a VIH/SIDA en adolescentes de un distrito de Lambayeque-Perú, 2015.

\section{Variable de Estudio}

Contacto sexual

$\begin{array}{lcc}\text { Sí } & 117 & 17,3 \\ \text { No } & 522 & 77,1 \\ \text { En blanco } & 38 & 5,6\end{array}$

Uso de protección

Nunca

$A$ veces

$33 \quad 28,2$

Casi siempre

Siempre

$42 \quad 6,2$

Contacto sexual con persona desconocida

$\begin{array}{lcc}\text { Sí } & 17 & 14,5 \\ \text { No } & 104 & 88,9\end{array}$

Usaría preservativo en contacto sexual con desconocido

$\begin{array}{lcc}\text { Sí } & 615 & 90,8 \\ \text { No } & 32 & 4,7 \\ \text { En blanco } & 30 & 4,4\end{array}$


disminuyen el contagio ( $p=0,04$ y $p=0,013)$, respectivamente. No se hallaron diferencias en prácticas sexuales referidas por tipo de colegio (Tabla 2).

Los hombres refirieron haber empezado a tener relaciones sexuales con anterioridad $(p=0,001)$, y siempre usaron algún tipo de protección $(p=0,001)$. Respondieron que usar preservativo $(p=0,001)$, evitar tener relaciones sexuales con prostitutas $(p=0,001)$ y evitar relaciones homosexuales $(p=0,001)$, disminuye el contagio de VIH. Por otro lado, las mujeres con mayor frecuencia definieron correctamente las siglas $\operatorname{SIDA}(p=0,003)$ y obtuvieron información por medio de amigos $(p=0,015)$ (Tabla 3$)$.

En el análisis por años de estudio, los de cuarto año obtuvieron mayor información de sus padres $(p=0,004)$ y amigos $(p=0,013)$. Los de quinto año refirieron tener mayores conocimientos $(p=0,001)$, obtuvieron información de las clases $(p=0,013)$ e internet $(p=0,001)$; además que el uso de preservativo $(p=0,001)$,

Tabla 3. Diferencias en conocimientos y percepciones según tipo de colegio, en adolescentes de un distrito de Lambayeque-Perú, 2015.

\begin{tabular}{llllll} 
Variable de estudio & \multicolumn{2}{c}{ Colegio público } & & \multicolumn{2}{c}{ Colegio privado } \\
\cline { 2 - 3 } & $\mathrm{N}$ & $\%$ & $\mathrm{~N}$ & $\%$ & $\begin{array}{c}\text { Valor } \\
\text { de } p\end{array}$
\end{tabular}

Fuente de información

Padres

Clases del colegio

Televisión

Amigos

Internet

En el colegio se habló sobre VIH/SIDA

Sí

No

EI SIDA tiene "cura"

Sí tiene cura

No tiene cura

No sé

Persona asintomática con VIH/SIDA puede contagiar

Sí

No

Vías de contagio

Picadura de mosquito.

Compartir utensilios de cocina

Pincharse con aguja utilizada

Recibir transfusión sanguínea

Relaciones sexuales con infectado

Beso en la boca

Uso de preservativo evita el contagio

Sí

No

Medidas preventivas

Uso de preservativo

Tener una sola pareja

Evitar tener relaciones sexuales

Evitar tener relaciones con prostitutas

Evitar tener relaciones homosexuales

Coitus interruptus

Percepción del nivel de conocimiento sobre VIH/SIDA

$$
\text { Alto }
$$

Medio

Bajo
156

277

143

62

195

338

75

76

282

54

260

151

66

53

312

336

356

45

56

356

236

255

130

277

237

44

50

285

79

\section{7,6}

66,7

34,5

14,9

46,9

81,4

18,1

18,3

67,9

13,0

62,6

36,4

15,9

12,8

75,2

80,9

85,8

10,8

13,5

85,8

56,9

61,4

31,3

66,7

57,1

10,6

12,1

68,7

19,0
99

184

116

46

107

228

33

87,0

12,6

28

10,7

180

50

68,7

19,0

169

88

64,5

33,6

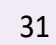

22

208

212

245

28

11,8

8,4

79,4

80,9

93,5

10,7

13,4

86,6

227

227

158

60,3

0,159

187

71,4

0,013

112

42,7

62,2

0,004

163

128

48,8

0,108

40

15,3

0,023

0,061 
tener una sola pareja $(p=0,01)$, y evitar tener relaciones homosexuales $(p=0,001)$ son formas de protección.

Se realizó un análisis multivariado de las preguntas 16: ¿Alguna vez en tu vida has tenido relaciones sexuales?, 17. Durante tus relaciones sexuales, ¿usas algún tipo de protección (preservativo, condón) contra el VIH?: 18: Alguna vez en tu vida has tenido relaciones sexuales con una persona desconocida? y 19: Si tuvieras un encuentro sexual con una persona desconocida ¿Utilizarías algún tipo de protección (condón)?, para determinar si existe asociación con el sexo, la edad, el tipo de colegio y el año de estudios, hallándose que para la primera, el sexo masculino ( $R P=1,2$ [IC 95\%=1,1-1,27]; $p=<0,001)$ y la edad $(p<0,001)$ se asocian a haber tenido relaciones sexuales. Para la pregunta 17 , se halló que los alumnos de colegio privado, usan 1,2 veces (IC 95\%=1,0-1,5; $p=0,017$ ) preservativo que los de público.

Para la pregunta 18 , los hombres tuvieron 5,1 veces la probabilidad de haber tenido relaciones con desconocidos (RP= 5,1 [IC 95\%=0,7-34,9]; $p=0,01$ ). En relación a la pregunta 19, no se halló asociación con ninguna variable.

\section{DISCUSIÓN}

Gran parte de los entrevistados no sabían el significado de las siglas VIH y SIDA, sin embargo, es discutible la importancia de este hallazgo, ya que las prácticas sexuales tienen poca relación con este conocimiento.

La mayoría respondió que la enfermedad no tiene cura. Si bien es cierto, la terapia antirretroviral reduce la carga viral a niveles indetectables, existen depósitos persistentes del virus en el organismo, siendo este el principal motivo para no definir una cura completa ${ }^{[8]}$. En el estudio de Dávila et al. ${ }^{[9]}$, en Venezuela en el año 2008, los estudiantes también respondieron que "no hay cura para la infección" (66,3\%). Esta concepción puede tener un impacto positivo en los estudiantes, disminuyendo los contactos sexuales de riesgo.

El $71,3 \%$ de los estudiantes creen tener un nivel de conocimientos intermedio, resultado similar al de Leguía et al. ${ }^{[6]}$ en Chiclayo en el 2011 (75,8\%), y mayor al de Quispe ${ }^{[10]}$ en Lima-Perú en el 2006 y Catacora et al. ${ }^{[11]}$ en Tacna Perú en el 2004, sin embargo, la percepción no es igual al nivel de conocimientos, como se muestra en el estudio de Leguía et al. ${ }^{[6]}$ (75,8\% vs $22,7 \%$ respectivamente). Al no contar con un instrumento validado, no se pudo demostrar esto en el estudio.

Las fuentes de información más reportadas fueron las clases en el colegio, seguidas de la Internet y la televisión, hallazgos similares a los de Leguía et al. ${ }^{[6]}$ en Perú en el 2011, donde el 52,6\% de los alumnos mencionaron que sus conocimientos fueron adquiridos en el colegio. Actualmente, el uso de las tecnologías de información y redes sociales por parte de adolescentes y jóvenes, son herramientas que han demostrado ser útiles para la prevención e información en relación a enfermedades como VIH
[12]; por lo que esta herramienta podría implementarse en nuestra localidad. Actualmente no existen estudios locales al respecto.

Las relaciones sexuales fueron la vía de contagio más mencionada $(88,7 \%)$, al igual que en los estudios de Dávila ${ }^{[9]}$ en Venezuela $(95,7 \%)$ y Leguía et al. ${ }^{[6]}$ en Chiclayo $(95,4 \%)$. Estos resultados sugieren que los adolescentes reconocen que existe riesgo de infectarse durante el acto sexual. Por otro lado, sólo 2,5\% refirieron que tendrían relaciones sexuales con desconocidos, pero de tenerlas, el 90,8\% usaría preservativo. Esto contrasta con el dato de que sólo el 13,4\% del total cree que el preservativo ofrece total protección. Es notorio el desconocimiento del riesgo real por parte de los adolescentes.

A diferencia del estudio de Dávila et al. ${ }^{[9]}(60,6 \%)$ y al de Díaz et al. ${ }^{[13]}$ en Colombia (62,9\%), en el nuestro, el $10,7 \%$ creía que la transmisión era posible al "besar en la boca a una persona infectada", sin embargo, 14,3\% respondió que la transmisión por picadura de mosquito era posible. Ello sugiere déficit de conocimiento que podría ser abordado durante las clases del colegio. Díaz et al. ${ }^{[13]}$ halló que el $51,3 \%$ consideraban peligroso "compartir alimentos o agua con una persona infectada" y 27,2\% creían era peligroso "beber en un vaso usado por personas seropositivas". En nuestro estudio esto ocurrió en $11,1 \%$ de los encuestados.

Por otro lado, las medidas preventivas más referidas fueron: tener una sola pareja sexual, evitar relaciones sexuales con prostitutas y el uso de preservativo; estos resultados son parcialmente diferentes a las recomendaciones de ONUSIDA ${ }^{[3]}$, según esta organización, el aplazamiento del inicio de la actividad sexual, la abstinencia, mantenerse fiel a la pareja, la reducción de parejas sexuales y el uso correcto y sistemático del preservativo, reducen el riesgo de la exposición sexual al VIH. Según ENDES $2009^{[14]}$, la mayoría de los adolescentes conocen de la existencia de algún tipo de método anticonceptivo (98,3\%), sin embargo solo el $63,7 \%$ de los sexualmente activos utiliza alguno. En nuestro estudio, $71,2 \%$ refiere que el preservativo no los protege totalmente, sin embargo un $13,4 \%$ indicaron que la protección es total; esta información no se encuentra acorde con la evidencia científica actual, en donde revisiones sistemáticas como la de Weller et al. ${ }^{[15]}$ hallaron que el uso de preservativo reduce en un $80 \%$ la incidencia de VIH.

Aun así, las otras estrategias han demostrado ser más eficaces en la prevención de contagio ${ }^{[16]}$; pero tienen la gran desventaja de ser difíciles de implementar y requerir un abordaje más holístico del problema en los adolescentes; sin embargo, creemos que la implementación de programas de tutoría podría ayudar a sobrellevar estas dificultades. Se deben buscar estrategias para enfatizar estas estrategias en jóvenes y adolescentes, pues no todos tienen conocimientos suficientes acerca de ellas; por ejemplo, sólo 35,7\% estuvieron de acuerdo en que la abstinencia es una medida recomendable.

En cuanto a prácticas, solo el 22,9\% de los encuestados habían tenido contacto sexual. Este dato puede ser un sesgo de 
información, pues según la Encuesta Nacional de la Juventud en el $2011^{[17]}, 72,3 \%$ de los adolescentes ya han iniciado su vida sexual. Solo el 6,2\% refieren usar siempre preservativo.

Las mujeres refirieron mayor información proveniente de amigos $(p=0,015)$, lo que nos habla de la influencia que tiene el entorno social en ellas y tal vez una mayor frecuencia de amistades del sexo opuesto; mientras que los hombres muestran un mayor conocimiento de las vías de contagio. Esto difiere a lo evidenciado por estudios como los de Díaz et al. ${ }^{[13]} \mathrm{y}$ de Rengifo ${ }^{[18]}$, realizados en Colombia. Lo hallado en nuestro estudio puede deberse a que los hombres sienten mayor interés en temas de sexualidad, ya que son ellos quienes inician antes su vida sexual y se informan más sobre sobre situaciones de riesgo ante la infección por VIH.

En los alumnos de quinto año, la principal fuente de información fueron las clases del colegio; lo que puede explicarse debido a haber recibido por más años educación sexual en el colegio, en el marco de la estrategia conjunta de educación sexual integral, del Ministerio de Educación y el Ministerio de Salud implementada desde el $2008{ }^{[19]}$. Sin embargo, puede haber sesgo de información, pues otros estudios mencionan que la televisión y la Internet son las principales fuentes. Castillo et al. ${ }^{[20]}$ en España, halló que las principales fuentes de información fueron, televisión, prensa y radio.

Los adolescentes de colegio público respondieron que evitar relaciones con homosexuales es una medida preventiva adecuada $(p=0,023)$, a diferencia de los colegios privados, en los que los entrevistados respondieron que tener una sola pareja sexual y la abstinencia, son las medidas más recomendables. Según estos resultados, los de colegio público tendrían una mayor conducta de riesgo. Gómez et al. ${ }^{[21]}$ en Colombia, halló que estudiar en un colegio privado se asocia a un buen conocimiento en $\mathrm{VIH} /$ SIDA. No se ha explorado la posible asociación entre religión, conocimientos, percepciones y conducta sexual ni tampoco la influencia del nivel socio-económico, el uso de tabaco y alcohol que han sido asociadas a la conducta sexual.

En las limitaciones, debemos mencionar que el cuestionario tiene validación de expertos y prueba piloto, más no se determinó la confiabilidad. Sin embargo, creemos que es una herramienta útil y rápida para el tamizaje de conocimientos y prácticas referidas. El tema es complejo y se sugiere su abordaje por medio de investigación cualitativa con entrevistas a profundidad.

Se recomienda seguir con la implementación educativa y darle mayor valor en las aulas al tema de VIH/SIDA, para que aquellos que aún no tienen algunos conceptos e ideas claras, puedan informarse, y para quienes tienen conocimientos previos, aclararen dudas. Asimismo, esperamos que surjan más investigaciones en el medio, para poder contrastar resultados y ver cambios a futuro.

Finalmente, según lo hallado en este estudio, el conocimiento relacionado a VIH/SIDA es parcial y para mejorar las prácticas, es necesario el trabajo conjunto entre profesores, padres, alumnos y el apoyo del gobierno. Este problema debe enfocarse considerando al adolescente como persona, teniendo en cuenta su antropología y necesidades. El abordaje biológico y reproductivo es insuficiente, siendo necesario incluir el aspecto psicológico y emocional, además de asegurarse de que la información sea entendida, aceptada y finalmente practicada.

En conclusión, la mayoría de los estudiantes del tercer, cuarto y quinto año de educación secundaria, del distrito de FerreñafeLambayeque, creen tener nivel intermedio de conocimientos sobre VIH/SIDA, siendo la principal fuente de obtención de información fue las clases en el colegio. Las vías de contagio más mencionadas fueron las relaciones sexuales y las medidas preventivas más referidas fueron el tener una sola pareja sexual, evitar relaciones sexuales con prostitutas y el uso de preservativo. Los hombres muestran mayor conocimiento de las vías de contagio y los alumnos de quinto año de secundaria refieren mayor porcentaje de relaciones sexuales, en comparación a los demás años de estudio. Finalmente, los alumnos de colegios públicos tienen mayor conocimiento sobre factores de riesgo y los de colegios privados sobre medidas preventivas.

Contribuciones de autoría: Juan William Baca Sánchez y Claudia Hidalgo Palacios han participado en la concepción, diseño, recolección, obtención, análisis, interpretación de datos, redacción del artículo, obtención de financiamiento y material de estudio. Además Franco Ernesto León Jiménez participó en la concepción, realizó análisis e interpretación de datos, revisión crítica del artículo, aprobación de su versión final y asesoría estadística; y Nancy Malca Tello contribuyó en la redacción y asesoría técnica del artículo.

Fuentes de financiamiento: Autofinanciado.

Conflictos de interés: Franco León Jiménez es miembro del CEI que aprobó la ejecución del estudio.

\section{REFERENCIAS BIBLIOGRÁFICAS}

1. Dirección General Epidemiología, Ministerio de Salud del Perú. $1^{\circ}$ de Diciembre: Día Mundial de Lucha Contra el VIH-SIDA. Boletín Epidemiológico [Internet]. 2013 [citado 15 Set 2015];(49):1013-30. Disponible en: http://www.dge.gob.pe/portal/docs/vigilancia/ boletines/2013/49.pdf

2. Dirección General Epidemiología, Ministerio de Salud del Perú. Análisis de la Situación Epidemiológica del VIH/SIDA en el Perú 2013. Lima, Perú: Ministerio de Salud; 2013.

3. ONUSIDA. Informe sobre la epidemia mundial de sida 2004. Suiza: Organización Mundial de la Salud; 2004.

4. Ministerio de Salud del Perú. Población Estimada por Edades Simples y Grupos de Edad, según Provincia y Distrito, Departamento de Lambayeque-2015. Lima, Perú: Ministerio de Salud; 2015.

5. Ministerio de Salud del Perú. Encuesta global de salud escolar. Resultados - Perú 2010. Lima, Perú: Ministerio de Salud; 2011.

6. Leguía J, Niño B, Perales J, Díaz C. Nivel de conocimientos sobre VIHSIDA en una Institución Educativa de José Leonardo Ortiz-Chiclayo. Marzo-Mayo 2011. Rev Cuerpo Med HNAAA. 2012;5(4):37-41. 
7. Torres T, Reynaldos C, Lozano A, Munquía J. Cultural Conceptions of HIV/AIDS among teenagers in Bolivia, Chile and Mexico. Rev Saude Publica. 2010;44(5):820-9.

8. Malatinkova E, De Spiegelaere W, Bonczkowski P, Kiselinova M, Vervisch K, Trypsteen W, et al. Impact of a decade of successful antiretroviral therapy initiated at HIV-1 seroconversion on blood and rectal reservoirs. Elife. 2015;4:e09115. doi: 10.7554/ elife.09115..

9. Dávila M, Tagliaferro A, Bullones X, Daza D. Nivel de Conocimiento de Adolescentes sobre VIH/SIDA. Rev Salud Pública. 2008;10(5):71622.

10. Quispe R. Nivel de Conocimiento acerca de las formas de Transmisión del VIH/SIDA y las actitudes hacia las prácticas sexuales riesgosas de los adolescentes de la Institución Educativa Isaías Ardiles del distrito de Pachacámac. Julio 2005 - Enero 2006 [tesis]. Lima, Perú: Facultad de Medicina Humana, Universidad Nacional Mayor de San Marcos; 2006.

11. Catacora F, Villanueva J. Conocimientos sobre VIH/SIDA y prácticas sexuales de escolares de Tacna, Perú 2004. Rev Peru Med Exp Salud Publica. 2007;24(3):240-7.

12. Hightow-Weidman LB, Muessig KE, Bauermeister J, Zhang C, LeGrand S. Youth, technology, and HIV: recent advances and future directions. Curr HIV/AIDS Rep. 2015;12(4):500-15.

13. Díaz C, Cantillo L, García K, Martínez L, Vega J. Conocimientos sobre VIH/SIDA en Adolescentes de una Universidad de CartagenaColombia, 2011. Hacia Promoc Salud. 2014;19(2):38-52.

14. Instituto Nacional de Estadística e Informática. ENDES 2009 Informe Principal [Internet]. Lima, Perú: INEI; c2015 [citado 20
Ago 2015]. Disponible en: http://proyectos.inei.gob.pe/endes/ endes2009/resultados/

15. Weller SC, Davis-Beaty K. Efectividad del preservativo en la reducción de la transmisión del VIH en heterosexuales. Cochrane Database of Systematic Reviews 2002, Issue 1. Art. No.: CD003255. DOI: 10.1002/14651858.CD003255

16. National Institute of Allergy and Infectious Diseases. Workshop Summary: Evidence on Condom Effectiveness for Sexually Transmitted Disease (STD) Prevention. June 12-13, 2000. [Internet]. Herndon, Virginia: NIH; 2001 [citado 24 Nov 2015]. Disponible en: https://chastity.com/wp/wp-content/uploads/2013/05/NIHCondom-Report.pdf

17. Ministerio de Educación del Perú. Encuesta Nacional de la Juventud 2011 [Internet]. Lima, Perú: MINEDU; 2012 [citado 25 Nov 2015] Disponible en: http://www.unfpa.org.pe/publicaciones/ publicacionesperu/SENAJU-INEI-ENAJUV-2011.pdf

18. Rengifo H. Conocimientos y prácticas en salud sexual y reproductiva de adolescentes escolares en un municipio colombiano. Revista Salud Pública. 2012;14(4):558-69.

19. Ministerio de Educación del Perú. Educación Sexual Integral en los Colegios [Internet]. Lima, Perú: Oficina de Prensa-MINEDU; 2010 [citado 16 Ago 2015]. Disponible en: http://www.minedu.gob.pe/ noticias/index.php?id=10421

20. Castillo MD, Gil E, León M, Naranjo JA. Conocimiento de los escolares sobre el SIDA. Revista de SEMG. 2003;51:109-20.

21. Gomez E, Cogollo Z. Conocimiento sobre VIH-SIDA en estudiantes de secundaria de Cartagena, Colombia. Rev Salud Pública. 2011;13(5):778-84.

\title{
Ahora puede enviar sus artículos para Acta Médica Peruana en nuestro Open Journal System:
}

\author{
www.amp.cmp.org.pe
}

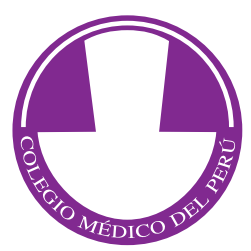

